\title{
Zoom Based Super-Resolution: A Fast Approach Using Particle Swarm Optimization
}

\author{
Prakash Gajjar and Manjunath Joshi \\ Dhirubhai Ambani Institute of Information and Communication Technology, \\ Gandhinagar, India \\ \{prakash_gajjar,mv_joshi\}@daiict.ac.in
}

\begin{abstract}
Given a set of images captured using different integer zoom settings of a camera, we propose a fast approach to obtain superresolution (SR) for the least zoomed image at a resolution of the most zoomed image. We first obtain SR approximation to the super-resolved image using a learning based approach that uses training database consisting of low-resolution (LR) and their high-resolution (HR) images. We model the LR observations as the aliased and noisy versions of their HR parts and estimate the decimation using the learned SR approximation and the available least zoomed observation. A discontinuity preserving Markov random field (MRF) is used as a prior and its parameters are estimated using the SR approximation. Finally Maximum a posteriori (MAP)-MRF formulation is used and the final cost function is optimized using particle swarm optimization (PSO) technique which is computationally efficient compared to simulated annealing. The proposed method can be used in multiresolution fusion for remotely sensed images where the available HR panchromatic image can be used to obtain HR multispectral images. Another interesting application is in immersive navigation for walk through application. Here one can change zoom setting without compromising on the spatial resolution.
\end{abstract}

Keywords: particle swarm optimization, learning, wavelet.

\section{Introduction}

Super-resolution refers to an algorithmic approach to obtain high resolution image from one or more low-resolution observations. Several approaches to the SR problem have been proposed. These include motion-based approaches [104], motion free approaches [39] and example-based approaches [521. Our work in this paper is derived from the work reported in [3]. Here the authors superresolve the least zoomed image consisting of the entire scene using a set of differently zoomed observations. A model based approach was used where the minimization was carried out using computationally taxing simulated annealing (SA) optimization tool. A fixed aliasing matrix was used and the MRF prior model parameters were adjusted by trial and error approach. In this work, we

A. Elmoataz et al. (Eds.): ICISP 2010, LNCS 6134, pp. 63-70, 2010.

(C) Springer-Verlag Berlin Heidelberg 2010 
first obtain a close approximation to the super-resolved image by using a learning based approach and estimate both the aliasing and the discontinuity preserving MRF prior parameters using this approximation. The assumption is that these estimated parameters are close to true parameters. Finally, a computationally efficient PSO optimization is used to minimize the cost function. The authors in [6] develop methods for constructing a single still image called 'salient still' from a video which includes variations in focal length or field of view, or moving objects. However our work is different from theirs. The main highlights of our work are, (1) We obtain a close approximation to final super-resolved image using a learning based technique. This is done since we do not have the original HR image and it has to be estimated, (2) We estimate the decimation matrix from the close SR approximation and the zoomed observations, (3) The HR image is modeled as a discontinuity preserving MRF prior and the model parameters are estimated using the SR approximation and (4) The non-convex cost function is minimized using the computationally efficient PSO technique.

\section{Problem Formulation}

Let $\left\{Y_{i}\right\}_{i=1}^{p}$ be a set consisting of $p$ low resolution observed images each of which is obtained by adjusting the optical zoom. The size of each observation is $M \times M$ pixels. The relationship between these low resolution images and the high resolution image is shown in Fig.1. Here the most zoomed image of the scene $Y_{3}$ has the highest resolution. The least zoomed image $Y_{1}$, corresponding to the entire scene, needs to be upsampled to the size of $\left(l_{1} l_{2} \cdots l_{p-1}\right) \times(M \times M)=$ $(N \times N)$ pixels, where $l_{1}, l_{2}, \ldots, l_{p-1}$ are the zoom factors between observed images of scene $Y_{1} Y_{2}, Y_{2} Y_{3}, \ldots, Y_{(p-1)} Y_{p}$, respectively. Given $Y_{p}$, the remaining $(p-1)$ observed images can be modeled as aliased and noisy versions of this single high-resolution image $(Z)$ of appropriate region. Note that although the observations are of same size, they are at different spatial resolutions. We write the forward model for the observed images as expressed in [3].

$$
\underline{\mathbf{y}}_{m}=D_{m} C_{m} \underline{\mathbf{z}}+\underline{\mathbf{n}}_{m},
$$

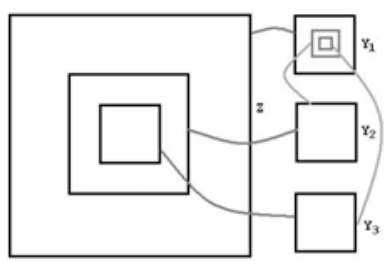

Fig. 1. Illustration of observations at different zoom levels. Here $Z$ is the superresolved image, $Y_{1}$ and $Y_{3}$ are the least zoomed and the most zoomed observations, respectively. 
where $m=1,2, \ldots, p$. Here $\underline{\mathbf{y}}, \underline{\mathbf{z}}$ and $\underline{\mathbf{n}}$ are vector representation of the observed image, super-resolved image and noise respectively. $C$ is cropping operator and $D$ is aliasing matrix. The noise $\mathbf{n}$ is assumed to be independent and identically distributed with zero mean and variance $\sigma_{n}^{2}$. Now the problem is to estimate $\underline{\mathbf{z}}$ given $\underline{\mathbf{y}}_{m} \mathrm{~s}$ which is an ill-posed problem and requires proper regularization.

\section{Proposed Approach}

\subsection{Learning the SR Approximation}

We first obtain the close approximation of the super-resolved image using a learning based method and use this for alias estimation as well as for prior parameter estimation. The learning based method requires the use of a database of sets of LR-HR images. This database is constructed using a high resolution camera. Once this database is ready it can be used later with no extra cost as it is one time operation. Each set consists of a LR image of size $M \times M$ and its HR versions of size $2 M \times 2 M, 4 M \times 4 M$ and so on. The learning technique for a decimation factor of $2(q=2)$ is as follows.

Step 1: Compute two level wavelet transform of the test image and each LR training image. Compute one level wavelet transform of each HR training image (see Fig:2).

Step 2: Obtain best matching LR training image for each location $(i, j)$ in subband 0 of the test image using minimum sum of absolute difference between a total of 15 wavelet coefficients at $(i, j+M / 4)$ in subbands $I,(i+M / 4, j)$ in subband $I I,(i+M / 4, j+M / 4)$ in subbands $I I I$ and corresponding $2 \times 2$ blocks in subabands $I V-V I$ of the test image and those of LR training images.

Step 3: Copy the corresponding $4 \times 4$ blocks of wavelet coefficients in subbands $I-I I I$ of the HR version of the best matching LR training image to the subbands $V I I-I X$ of the test image.

Step 4: Take inverse wavelet transform of the reconstructed transform of the test image to get high spatial resolution image.

For the test image $Y_{1}$, we obtain the SR approximation of size $2 M \times 2 M$ using training images of size $M \times M$ and $2 M \times 2 M$. We then use this SR approximation as a test image and obtain the SR approximation of size $4 M \times 4 M$ using training images of size $2 M \times 2 M$ and $4 M \times 4 M$. Similarly, we learn the SR approximation of size $2 M \times 2 M$ for the test image $Y_{2}$ and replace its centermost $M \times M$ region by the most zoomed observation $Y_{3}$ as it is captured at the highest resolution. We then insert this image in the centermost $2 M \times 2 M$ part of the SR approximation of $Y_{1}$ having size $4 M \times 4 M$ and use resulting image as an SR approximation to the super-resolved image. 

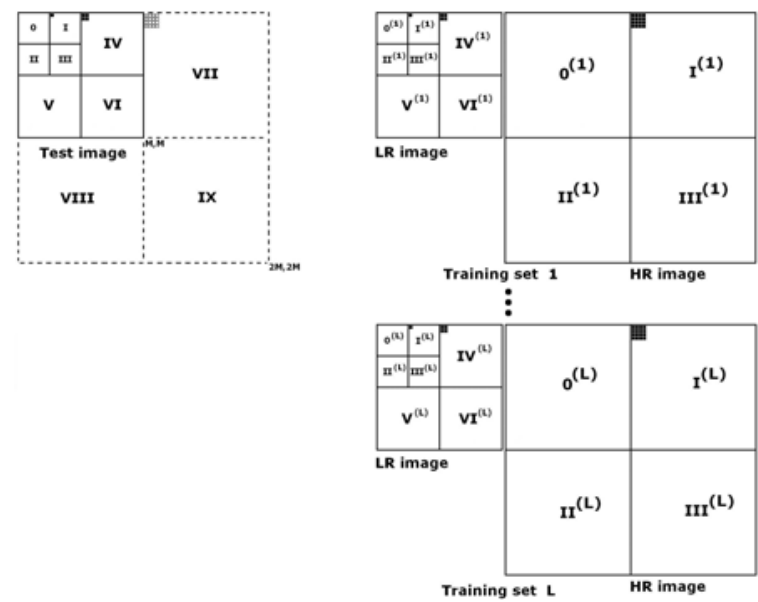

Fig. 2. Wavelet decomposition for learning finer details from database of LR-HR images

\subsection{Decimation Estimation}

Generally, the decimation model to obtain the aliased pixel intensities from the high resolution pixels, for a decimation factor of $q$, has the form

$$
D=\left(\begin{array}{ccccc}
a_{1} a_{2} \ldots & a_{q^{2}} & & 0 \\
& & a_{1} a_{2} \ldots & a_{q^{2}} & \\
& & & & \\
0 & & & a_{1} a_{2} \ldots & a_{q^{2}}
\end{array}\right) .
$$

The decimation matrix with entries $a_{i}=\frac{1}{q^{2}}, i=1,2, \ldots q^{2}$ in (2) indicates that a low resolution pixel intensity $y(i, j)$ is obtained by averaging the intensities of $q^{2}$ pixels corresponding to the same scene in the high resolution image and adding noise intensity $n(i, j)[10$. Due to different configurations of camera hardware and different zoom settings, the aliasing differs from image to image. Hence the aliasing has to be estimated. Since the SR approximation is already available as discussed in Section 3.1. we make use of the same to obtain the decimation matrix entries and thus learn the aliasing better. The estimated decimation matrix implicitly captures the space invariant image blur in the downsampling process. We use a simple least squares approach to estimate the decimation matrix entries $a_{i}$.

\subsection{Image Field Modeling and Super-Resolving the Scene}

In real world images, the change in intensities is usually gradual. This local dependencies among the pixels can be represented using MRF model prior. This constraint pushes the reconstruction towards smooth entity and causes the distortions along discontinuities. We include binary line fields $L$ and $V$ to preserve 
the discontinuities while smoothing the edges. The element $l_{i, j}$ and $v_{i, j}$ of these fields at location $(i, j)$ are set to 1 if there exists horizontal or vertical discontinuity respectively at location $(i, j)$. The energy function for $Z$ with discontinuity fields $L$ and $V$ is given as, $V(\underline{\mathbf{z}})=\sum_{i, j} \mu\left[\left(z_{i, j}-z_{i, j-1}\right)^{2}\left(1-v_{i, j}\right)+\left(z_{i, j+1}-\right.\right.$ $\left.\left.z_{i, j}\right)^{2}\left(1-v_{i, j+1}\right)+\left(z_{i, j}-z_{i-1, j}\right)^{2}\left(1-l_{i, j}\right)+\left(z_{i+1, j}-z_{i, j}\right)^{2}\left(1-l_{i+1, j}\right)\right]+\gamma\left[v_{i, j}+\right.$ $\left.v_{i, j+1}+l_{i, j}+l_{i+1, j}\right]$. This energy function consists of the smoothness term and the penalty term to prevent spurious discontinuities. Here $\mu$ and $\gamma$ are the model parameters. We estimate these parameters from the SR approximation using homotopy continuation method proposed in [12].

Using the data fitting term (11) and the energy function $V(\underline{\mathbf{z}})$ and using the MAP-MRF formulation the estimate of the super-resolved image can be given as,

$$
\hat{\mathbf{z}}=\underset{\underline{\mathbf{z}}}{\operatorname{argmin}}\left[\sum_{m=1}^{p} \frac{\left\|\underline{\mathbf{y}}_{m}-D_{m} C_{m} \underline{\mathbf{z}}\right\|^{2}}{2 \sigma_{n}^{2}}+V(\underline{\mathbf{z}})\right] .
$$

This cost function is non convex and non regular hence cannot be minimized using simple differentiation based optimization techniques or using graph-cuts. This motivates us to use PSO which can be implemented to carry out parallel operations in order to reduce the computation time.

\subsection{Particle Swarm Optimization}

Global optimization techniques such as simulated annealing, graph-cuts are widely used for optimization of non-convex cost function. The SA technique is computationally taxing and takes a very long time for convergence. It may be mentioned that many of the researchers have attempted to use graph-cuts for minimization of cost functions of binary variables. The energy function derived in the previous section cannot be optimized using graph-cuts techniques directly. A variant of graph-cuts, quadratic pseudo-boolean optimization technique can be used for minimizing functions with both submodular and non-submodular terms [15]. It requires approximation to the cost function and leads to the solution that differs much from the true solution. We propose the use of particle swarm optimization technique [13] that speeds up the convergence and reduces the computation time. PSO is a heuristic approach and is initialized with a group of particles. The algorithm then searches for the best solution through an iterative process. At every iteration, the fitness of each particle is evaluated using the fitness (cost) function. If it is the best value the particle has achieved so far, the particle stores that value as 'personal best'. The best fitness value achieved by any particle during current iteration is stored as 'global best'. Let $S=\left\{Z_{b} \mid b=1,2, \ldots, B\right\}$ be a swarm initialized with initial HR images as the particles. The fitness of the particle $Z_{b}$ at $n^{t h}$ iteration is denoted by $F_{Z_{b}}^{n}$. The fitness function in our case is the cost function that has to be minimized. Let $Z_{b p}^{n}$ be the personal best of particle $Z_{b}$ and $Z_{g}^{n}$ be the group best at the $n^{\text {th }}$ iteration. The fitness values of $Z_{b p}^{n}$ and $Z_{g}^{n}$ are denoted by $F_{Z_{b p}^{n}}$ and $F_{Z_{g}^{n}}$, respectively. While using the PSO the velocity $V_{b}$ and position $Z_{b}$ after $n^{\text {th }}$ iteration are updated according to $V_{b}^{n+1}=w V_{b}^{n}+c_{1} r_{1}\left(F_{Z_{b p}^{n}}-F_{Z_{b}}^{n}\right)+c_{2} r_{2}\left(F_{Z_{g}^{n}}-F_{Z_{b}}^{n}\right)$ and 
$Z_{b}^{n+1}=Z_{b}^{n}+V_{b}^{n}$ [14. Here $w$ is weighting function, $r_{1}$ and $r_{2}$ are random numbers uniformly distributed in $[0,1], c_{1}$ and $c_{2}$ are cognitive and social parameters, respectively. It may be noted here that the evaluation of the fitness of a particle does not depend on that of the other particles. Hence this concurrent process can be implemented using parallel processors and the optimization time can be reduced further. Recently many variations of PSO have been proposed for fast convergence [117].

\section{Experimental Results}

We conducted experiments on real images captured by adjusting the zoom setting of a camera. The observed images $Y_{1}, Y_{2}$ and $Y_{3}$ are shown in Fig 3 (a)-(c). The zoom factor between $Y_{1}$ and $Y_{2}$ is 2 that between $Y_{1}$ and $Y_{3}$ is 4 . All observations are of size $64 \times 64$ pixels. The estimated values of the model parameter $\mu$ for images 1, 2 and 3 are 0.02057, 0.01337 and 0.00913, respectively. Similarly, the values of $\gamma$ for these images are 4.2863, 2.7917 and 3.1809, respectively. These are approximated using the HR approximation. PSO parameters are chosen as $c_{1}=1, c_{2}=0.5$ and $w=1$. We expand the least zoomed images using existing interpolation techniques and use them as particles in PSO. In our experiment, the swarm consists of 10 particles. Three particles are obtained using bilinear, bicubic and Lanczose interpolation techniques. The initial SR approximation is also used as one of the particles. The remaining particles are obtained by manipulating these four particles. We compare the results with the zoom based approach proposed in [3]. Since zoom based SR problem is not addressed by many researchers, we compare our results with the recent SR technique proposed in [2. For quantitative comparison, we use peak signal to noise ratio (PSNR) given by $10 \log \frac{255^{2}}{M N \sum_{i, j}[f(i, j)-\hat{f}(i, j)]^{2}}$, where $f(i, j)$ is the original high resolution image of size $M \times N$ and $\hat{f}(i, j)$ is interpolated or super-resolved image.

The results of our experiments for decimation factor $q=4$ are shown in Fig 3. Fig 3(d) shows Image 1, Image 2 and Image 3 expanded using bicubic interpolation technique. Fig 3(e) and (f) show the images super-resolved using the algorithm proposed in [2] and [3], respectively. Images super-resolved using the proposed approach using PSO are displayed in Fig $3(\mathrm{~g})$. Images obtained using bicubic interpolation are over-smooth while those shown in Fig. [3(e) and (f) lack high frequency details. It can be seen that the high frequency details are better preserved in images super-resolved using the proposed approach than those in the images obtained using other approaches. The text in Fig. 3(g) appears sharp and the rectangular boarder on the floor are thin indicating preservation of high frequency details (see image 3 in Fig.3(g)). Table 1 shows that the PSNR of the images super-resolved using proposed approach is comparable to those obtained using the other approaches. Although there is a slight decrease in PSNR for image 1 and image 3 , the computation time is much less for the proposed method. The optimization time using SA is almost around 4 hours, whereas that for the proposed method is less than half a minute. 

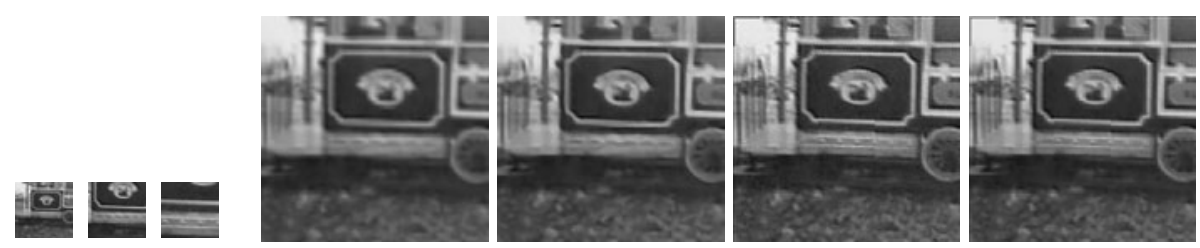

Image 1
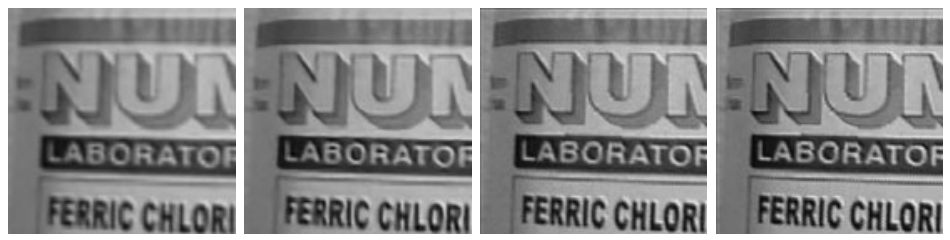

Image 2

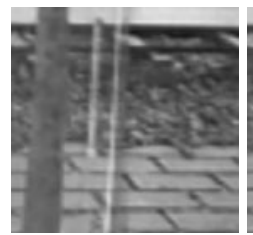

(d)

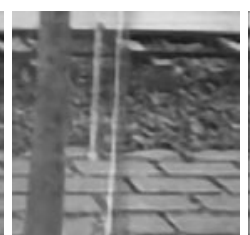

Image 3

(e)

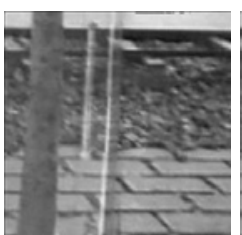

(f)

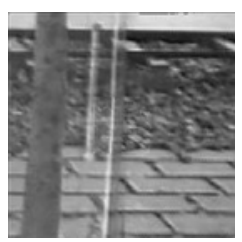

(g)

Fig. 3. Results for $q=4$. (a)-(c) Observations: (a) is the least zoomed observation and (c) is the most zoomed one, (d) image expanded using bicubic interpolation, (e) superresolved image using the approach proposed in [2, (f) super-resolved image using the approach proposed in [3] and (g) super-resolved image using proposed approach.

Table 1. Performance comparison

\begin{tabular}{|c|c|c|c|c|c|c|}
\hline Image & $\begin{array}{c}\text { Optimization Time } \\
\text { (in seconds) }\end{array}$ & \multicolumn{4}{|c|}{$\begin{array}{c}\text { Quantitative comparison } \\
\text { (PSNR in dB) }\end{array}$} \\
\cline { 2 - 7 } & SA & PSO & $\begin{array}{c}\text { Bicubic } \\
\text { interpolation }\end{array}$ & $\begin{array}{c}\text { Kim and Kwon } \\
\text { approach in [2 }\end{array}$ & $\begin{array}{c}\text { MRF with SA } \\
\text { in [3] }\end{array}$ & Proposed \\
\hline 1 & 16730.94 & 21.172 & 22.13 & 21.00 & 24.81 & 22.43 \\
\hline 2 & 15419.78 & 20.570 & 19.80 & 19.30 & 23.32 & 23.50 \\
\hline 3 & 14827.32 & 21.098 & 22.03 & 21.40 & 25.11 & 23.32 \\
\hline
\end{tabular}

\section{Conclusions}

We have proposed a fast approach for zoom based super-resolution. A learning based approach was used for obtaining a HR approximation and the MAP-MRF formulation was used to solve the problem. The aliasing matrix entries and the discontinuity preserving MRF parameters were estimated using the learned SR approximation. We used a computationally efficient PSO for cost minimization 
purposes and the time complexity of the same is much less compared to SA. The results indicate that the proposed method yields perceptually better superresolved images and offers a significant computational speed advantage.

\section{References}

1. Chan, T.M., Zhang, J.P., Pu, J., Huang, H.: Neighbor embedding based superresolution algorithm through edge detection and feature selection. Pattern Recognition Letters 30(5), 494-502 (2009)

2. Kim, K.I., Kwon, Y.: Example-based learning for single-image super-resolution. In: 30th annual symposium of the Deutsche Arbeitsgemeinschaft fur Mustererkennung, pp. 456-465 (2008)

3. Joshi, M.V., Chaudhuri, S., Rajkiran, P.: Superresolution imaging: use of zoom as a cue. Image and Vision Computing 14(22), 1185-1196 (2004)

4. Shen, H., Zhang, L., Huang, B., Li, P.: A MAP approach for joint motion estimation, segmentation, and super resolution. IEEE Trans. Image Process 2(16), 479-490 (2007)

5. Freeman, W.T., Jones, T.R., Pasztor, E.C.: Examplebased super-resolution. IEEE Computer Graphics and Applications 22(2), 56-65 (2002)

6. Teodosio, L., Bender, W.: Salient stills. ACM Transactions on Multimedia Computing, Communications and Applications 1(1), 16-36 (2005)

7. Doctor, S., Venayagamoorthy, G.K.: Improving the Performance of Particle Swarm Optimization using Adaptive Critics Designs. In: IEEE Swarm Intelligence Symposium, USA, pp. 1-4 (2005)

8. Joshi, M.V., Chaudhuri, S., Panuganti, R.: A learning based method for image super-resolution from zoomed observations. IEEE Trans. Systems, Man and Cybernetics, Part B, Special Issue on learning in computer vision and pattern recognition 35(3), 527-537 (2005)

9. Rajagopalan, A.N., Kiran, V.P.: Motion-free super-resolution and the role of relative blur. Journal of the Optical Society of America - A 20(11), 2022-2032 (2003)

10. Schultz, R.R., Stevenson, R.L.: A bayesian approach to image expansion for improved definition. IEEE Trans. Image Process 3(3), 233-242 (1994)

11. Chen, Q., Yang, J., Gou, J.: Image Compression Method Using Improved PSO Vector Quantization. In: Wang, L., Chen, K., S. Ong, Y. (eds.) ICNC 2005. LNCS, vol. 3612, pp. 490-495. Springer, Heidelberg (2005)

12. Nanda, P.K., Desai, U.B., Poonacha, P.G.: A homotopy continuation method for parameter estimation in MRF models and image restoration. In: IEEE sympo. on Circuits and Systems, vol. 3, pp. 273-276 (1994)

13. Kennedy, J., Eberhart, R.: Swarm intelligence. Morgan Kaufmann, San Francisco (2001)

14. Vrahatis, M.N., Parsopoulos, K.E.: Natural Computing. Kluwer, Dordrecht (2002)

15. Kolmogorov, V., Rother, C.: Minimizing non-submodular functions with graph cuts - a review. IEEE Trans. Pattern Analysis and Machine Intelligence 29(7), $1274-1279$ (2007) 